\title{
Centrifugal Force-Induced Elastic Field for a Stress-Free Annulus Made of Functionally Graded Polar Orthotropic Material from Circular Plates to Rings
}

\author{
By Vebil Yıldırım*
}

\begin{abstract}
Formation of a new kind of advanced material type from polar orthotropic materials obeying certain functional material grading rules has gained a great importance lately. In this study, a circular annulus rotating at a constant angular velocity and made of such kind of advanced materials having the same inhomogeneity index for both elasticity moduli and material density is studied analytically. Governing equation in terms of radial displacement is derived from the elasticity theory under axisymmetric conditions and plane stress assumption for such structures. After deriving closed-form solutions, effect of the aspect ratio (inner radius/outer radius) of a rotating circular annulus with uniform thickness on the dimensionless elastic field is investigated. It is observed that both the aspect ratio and inhomogeneity index of a simple power-law material grading rule have significant impacts on the linear elastic response arising out of rotation of the annulus at a constant angular velocity.
\end{abstract}

Keywords: Rotating discs, circular annulus, elasticity solution, functionally graded, polar orthotropic.

\section{Introduction}

Around 1970s, composite materials obtained by combining two or more materials at the macro-scale appeared in many engineering applications such as aircraft structures, automobile, transportation, marine, and building structures due to their high strength to weight and stiffness to weight ratios, high corrosion resistance, thermal and acoustic insulation properties, improved fatigue life, and good design practice than metallic structures (Gopalakrishnan et al. 2008, Campbell 2010). The fiber (reinforcement) and the matrix are two main constituents of a typical composite material. The reinforcement should be harder, stronger, and stiffer than the matrix. The smaller the diameter of the fiber is, the higher its strength. However the cost of the composite structure increases with decreasing diameter. The continuous phase of a composite material is called the matrix, which may be a polymer, metal, or ceramic. As a matrix material, polymers have low strength and stiffness as well as ductile while ceramics have high strength and stiffness but are brittle. As is well known, metals have moderate strength and stiffness together with considerably high ductility.

Composite materials are classified as fibrous composites, particulate composites and laminated composites. The fibrous composites, in which the matrix material has low stiffness, density and strength compared to the fibers or whiskers dispersed in a matrix. Carbon, graphite, E-glass etc. are commonly used for fibers while a polymeric material epoxy is used for the matrix material. Suspension of particles of one or more materials in a matrix of different material

*Professor, University of Çukurova, Turkey. 
forms a particulate composite. In a particulate composite both the matrix and particle materials may be metallic or non-metallic. Particulate composites tend to be much weaker and less stiff than continuous fiber composites, but they are usually much less expensive. The laminated composites consists of a number of laminas (plies) stacked together to form a composite structure. Each lamina contains fibers bounded by a matrix material are oriented in the same direction where the maximum strength is required. The most commonly used matrix material is epoxy resin while carbon, glass, aramid and boron are used for fiber materials. The lay-up in which the plies are stacked at different orientation angles is called a laminate. Unidirectional $\left(0^{\circ}\right)$ laminae are extremely strong and stiff in the $0^{\circ}$ direction. However, they are considerably weak in the $90^{\circ}$ direction. The laminated composites are orthotropic at the lamina level while they exhibit highly anisotropic properties at the laminate level.

From the composite materials, polar orthotropic materials were used to increase the critical speeds of rotating disks (Tang 1969, Murthy and Sherbourne 1970, Reddy and Srinath 1974, Ari-Gur and Stavsky 1981, Jain et al. 1999, 2000, Tütüncü 2000, Liang et al. 2002, Singh and Jain 2004, Çallığlu 2007, Çallığlu et al. 2005, 2006, Koo 2006, 2008, Gupta et al. 2006, Li et al. 2007, Eraslan et al. 2016, Y1ldırım 2018a-d). Murthy and Sherbourne (1970) presented analytical solutions for rotating anisotropic hollow discs with variable thickness for an annular disc (free-free) and a disc mounted on a circular rigid shaft (fixedfree). Reddy and Srinath (1974) obtained closed-form solutions for stresses and displacement in an anisotropic rotating circular disc of variable thickness and variable density. Çallığlu et al. (2006) investigated analytically the elastic-plastic stress analysis of a curvilinearly orthotropic rotating annular disc for strain hardening material behavior. Eraslan et al. (2016) developed an analytical solution in terms of hypergeometric functions to estimate the mechanical response of nonisothermal, orthotropic, variable thickness disks under a variety of boundary conditions such as rotating annular disks with two free surfaces, stationary annular disks with pressurized inner and free outer surfaces, and free inner and pressurized outer surfaces. Y1ldirım (2018a) introduced the complementary functions method (CFM) for the initial value problem solutions to the linear elastic analysis of anisotropic rotating uniform discs. The anisotropy effects are considered in this study for free-free, fixed-free, and fixed-guided rotating disks made of a polar orthotropic material.

As a new kind of advanced structural composite materials, functionally graded materials (FGMs) are originated in Japan (Mahamood et al. 2012, Mahamood and Akinlabi 2017). Mahamood and Akinlabi (2017) presented the concept of functionally graded materials as well as their use and different fabrication processes. A FGM consist of at least two constituents which are continuously graded along demanded engineered directions to meet the requirements of the applications. Unlike the classical composites, in a FGM structure inter-laminar failure due to the delamination along the interfaces of continuous and discontinuous plies does not occur. In a laminated composite structure considerable inter-laminar stresses may arise especially at the ends of discontinuous plies. Moreover inter-laminar failure may also occur when the inter- 
laminar stresses are much lower than the in-plane stresses. To sum up, functionally graded materials can withstand very high thermal gradient, can inhibit crack propagation, may provide thermal barrier, and also perfectly meet the requirements for minimizing thermo-mechanical mismatch in metal-ceramic bonding. Due to these impeccable advantages, functionally graded materials have attracted the attention of many researchers and industrial applications having severe operating conditions in the mineral processing industry along with machine, aerospace, energy, nuclear, defense, optoelectronics, dental, orthopedic, and automotive industries. Here some applications of FGMs are highlighted (Mahamood et al. 2012, Mahamood and Akinlabi 2017): linings for wear-resistant, abrasionresistant and impact-resistant, rocket heat shields, rocket engine components, heat exchanger tubes, thermoelectric generators, heat-engine components, plasma facings for fusion reactors, electrically insulating metal/ceramic joints, space plane bodies, teeth and bone replacements, penetration resistant materials used for armour plates and bullet-proof vests, protective coatings on turbine blades in gas turbine engines, graded refractive index materials, magnetic storage media, tribology, sensors, fire retardant doors, energy conversion devices, cutting tool insert coatings, automobile engine components, nuclear reactor components etc.

Low-cost ceramic-metal functionally graded materials are essentially used in FGM applications. They form an isotropic but inhomogeneous structure. In quest of searching more advanced materials, scholars have begun to apply anisotropic materials as functionally graded material constituents to form both anisotropic and inhomogeneous structures. For annular structures made of functionally graded anisotropic and inhomogeneous materials, there may be provided relatively a few studies on the stress and displacement analyses in the available literature (Gurushankar 1975, Mian and Spencer 1998, Durodola and Attia 2000, Chen et al. 2007, Wang and Sudak 2008, Zenkour 2009, Lubarda 2012, Peng and Li 2012, Boga 2016, Kacar and Yıldırım 2017, Zeng et al. 2017; Essa and Argeso 2017).

Gurushankar (1975) obtained closed form solution for the elastic fields in a rotationally symmetric, nonhomogeneous, polar orthotropic, annular disk of varying thickness and density, subjected to thermal loading. The variations of homogeneity, density and thickness are assumed to be hyperbolic in this study. Durodola and Attia (2000) studied with a functionally graded material which was modelled as a non-homogeneous orthotropic material for rotating hollow and uniform solid discs. Chen et al. (2007) studied analytically uniform rotating disc made of exponentially functionally graded materials with transverse isotropy. Lubarda (2012) worked on the elastic response of uniformly pressurized cylindrically anisotropic hollow uniform thin rotating discs. He used the finite difference method and a Fredholm integral equation. Peng and Li (2012) considered a functionally graded hollow polar-orthotropic rotating uniform disk for both power-law material grading rule and arbitrarily varying gradient. They also used Fredholm integral equation in the solution procedure. Kacar and Y1ldirım (2017) studied analytically the elastic behavior of a stress-free rotating circular uniform disc (a uniform annulus), a rotating uniform disc mounted a rigid shaft at its center, and a rotating disc attached a rigid shaft and guided at outer surface for the same issue for five types of materials. Zeng et al. (2017) considered 
variable thickness rotating discs made of a functionally graded fiber-reinforced material. Recently Essa and Argeso (2017) developed analytical solutions for the analysis of elastic polar orthotropic functionally graded annular disks rotating with constant angular velocity. Traction-free inner and outer surfaces, and annular disks mounted on a circular rigid shaft having traction-free outer surface were studied separately by Essa and Argeso (2017).

In the present study, an investigation of the variations of the elastic fields along the radial coordinate of a rotating circular annulus from the circular hollow plate to the thin rings is aimed. The annulus is assumed to be made of an anisotropic and nonhomogeneous material. To form such a kind of material, two anisotropic materials are gradually graded such that the mixture should obey a simple power material grading pattern. To conduct a parametric study, the same inhomogeneity index for both elasticity moduli and material density are employed. Closed-form solutions are obtained from the governing Navier equation under axisymmetric conditions, small displacements and plane stress assumption in the linear elasticity theory. Then a parametric study is conducted to study the effects of both the aspect ratio (inner radius/outer radius) and the inhomogeneity index of a rotating circular annulus with uniform thickness on the dimensionless elastic field.

\section{Exact Solutions}

Let's consider a disk made of a functionally graded polar orthotropic material. The disk is assumed to be very thin, as seen in Figure 1, so that in the z-direction, the stress and strain components are neglected, and shear stress is zero. By using the infinitesimal theory of elasticity with axisymmetric plain stress conditions, strain- displacement relations are written as follows

$$
\begin{aligned}
& \varepsilon_{r}(r)=u_{r}^{r}(r) \\
& \varepsilon_{\theta}(r)=\frac{u_{r}(r)}{r}
\end{aligned}
$$

Where $u_{r}$ is the radial displacement, $\varepsilon_{r}$ and $\varepsilon_{\theta}$ are the radial and tangential strain components, respectively. The prime symbol is used for the first derivative of the quantity with respect to the radial coordinate. Representing the radial and hoop stresses by $\sigma_{r}$ and $\sigma_{\theta}$, respectively, Hooke's law may be written in the following compact form of

$$
\begin{aligned}
& \sigma_{\mathrm{r}}(r)=C_{11}(r) \varepsilon_{r}(r)+C_{12}(r) \varepsilon_{\theta}(r) \\
& \sigma_{\theta}(r)=C_{12}(r) \varepsilon_{r}(r)+C_{22}(r) \varepsilon_{\theta}(r)
\end{aligned}
$$

By defining the following ratios

$$
\lambda_{1}=\frac{C_{12}(r)}{C_{11}(r)}=\lambda_{2} v_{r \theta}
$$


$\lambda_{2}=\frac{C_{22}(r)}{C_{11}(r)}=\frac{E_{\theta}(r)}{E_{r}(r)}$

and

$C_{o}=-\frac{E_{\theta}^{b} v_{r \theta}}{v_{\theta r}\left(v_{r \theta} v_{\theta r}-1\right)}$

material on-axis rigidities $\left(\theta=0^{\circ}\right)$ in Eq. (2) may be written as follows

$C_{11}(\mathrm{r})=\left(\frac{r}{b}\right)^{\beta} \mathrm{C}_{\mathrm{o}}$

$C_{12}(\mathrm{r})=\left(\frac{r}{b}\right)^{\beta} C_{o} \lambda_{1}$

$C_{22}(\mathrm{r})=\left(\frac{r}{b}\right)^{\beta} C_{o} \lambda_{2}$

Where Young`s modulus in the $r$ and $\theta$ directions are denoted by $E_{r}(r)$ and $E_{\theta}(r)$, the ratio $\lambda_{2}$ stands for the anisotropy/polar degree of the disc material, $\beta$ is the inhomogeneity index, $b$ is the outer radius and $E_{\theta}^{b}$ shows the reference value of Young's modulus of the mixture material located at the outer surface in the circumferential direction (Figure 1). For isotropic and homogeneous materials: $E=E_{\theta}=E_{r}$. In Equation (5) a simple power material grading rule is used. Poisson`s ratios in Equations (3) and (4), $v_{\theta r}$ and $v_{r \theta}$, which are assumed to be constant along the radial coordinate in the present study, are related by each other as follows

$\frac{v_{\theta r}}{E_{\theta}(r)}=\frac{v_{r \theta}}{E_{r}(r)}$

Figure 1. A Uniform Circular Annulus Rotating at a Constant Angular Velocity

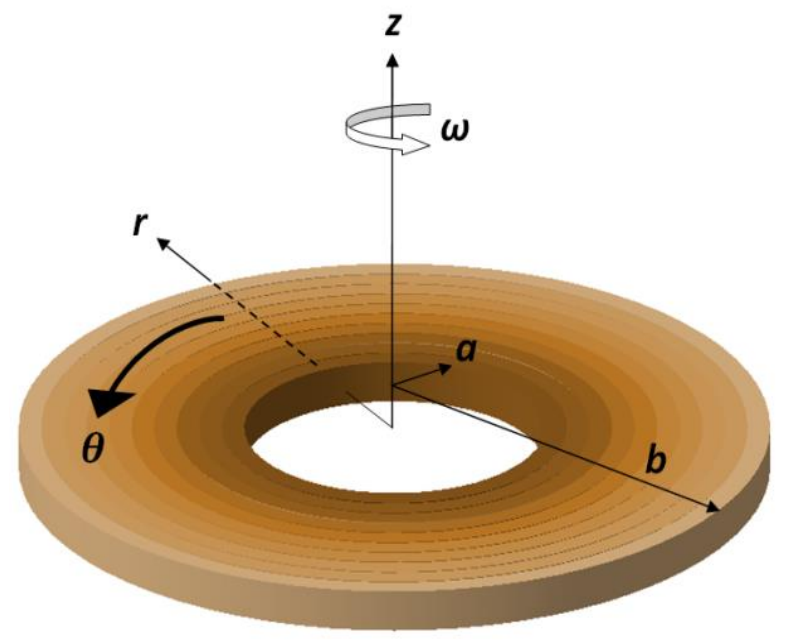


Equilibrium equation with centrifugal force effects is

$\sigma_{r}^{\prime}+\left(\frac{\sigma_{r}-\sigma_{\theta}}{r}\right)+\rho(r) \omega^{2} r=0$

Where $\rho(r)$ denotes the material density, which also radially varies with a simple power material grading rule, and $\omega$ is a constant angular velocity.

$\rho(\mathrm{r})=\left(\frac{r}{b}\right)^{\beta} \rho^{b}$

In the above $\rho^{b}$ stands for the reference value of the density of the mixture material located at the outer surface. Substitution stresses in Equation (2) and the first derivative of the radial stress with respect to the radial coordinate into the equilibrium equation, and then replacing strain components with their counterparts in terms of radial displacements, the following second order Navier differential equation is derived.

$$
u_{r}^{\prime \prime}+\left(\frac{1}{r}+\frac{C_{11}^{\prime}(r)}{C_{11}(r)}\right) u_{r}^{I}+\left(-\frac{\lambda_{2}}{r^{2}}+\frac{\lambda_{1}}{r} \frac{C_{11}^{\prime}(r)}{C_{11}(r)}\right) u_{r}=-\frac{\rho(r) \omega^{2} r}{C_{11}(r)}
$$

After implementation of the material grading rule given in Equations (5) and (8) into Equation (9), then Navier equation reduces to the following

$$
\frac{\left(\beta \lambda_{1}-\lambda_{2}\right)}{r^{2}} u_{r}+\frac{(1+\beta)}{r} u_{r}^{\prime}+u_{r}^{\prime \prime}=-\frac{r \omega^{2} \rho^{b}}{C_{0}}
$$

This equation is an Euler-Cauchy type differential equation with constant coefficients. General solution of Equation (10) will be in the form of

$u_{r}(\mathrm{r})=r^{\frac{1}{2}(-\beta-\xi)}\left(C_{1}+C_{2} r^{\xi}\right)+r^{3} \Omega$

Where $C_{1}$ and $C_{2}$ are integration constants, and

$$
\begin{aligned}
& \Omega=-\frac{\omega^{2} \rho^{b}}{C_{0}\left(9+3 \beta+\beta \lambda_{1}-\lambda_{2}\right)} \\
& \xi=\sqrt{\beta^{2}-4 \beta \lambda_{1}+4 \lambda_{2}}
\end{aligned}
$$

By using stress-displacement relations for a polar orthotropic material, the radial and hoop stresses may be written in terms of integration constant as follows 


$$
\begin{aligned}
& \sigma_{r}(r)=\frac{1}{2} r^{\frac{1}{2}(-2-\beta-\xi)}\left(-\left(-C_{2} r^{\xi}(-\beta+\xi)+C_{1}(\beta+\xi)-6 r^{\frac{1}{2}(6+\beta+\xi)} \Omega\right) C_{11}\right. \\
& \left.+2\left(C_{1}+C_{2} r^{\xi}+r^{\frac{1}{2}(6+\beta+\xi)} \Omega\right) C_{12}\right) \\
& \begin{array}{c}
\sigma_{\theta}(r)=\frac{1}{2} r^{\frac{1}{2}(-2-\beta-\xi)}\left(-\left(-C_{2} r^{\xi}(-\beta+\xi)+C_{1}(\beta+\xi)-6 r^{\frac{1}{2}(6+\beta+\xi)} \Omega\right) C_{12}\right. \\
\left.+2\left(C_{1}+C_{2} r^{\xi}+r^{\frac{1}{2}(6+\beta+\xi)} \Omega\right) C_{22}\right)
\end{array}
\end{aligned}
$$

By employing stress-free boundary conditions, $\sigma_{r}(a)=0$ and $\sigma_{r}(b)=0$, unknown coefficients in Equations (11) and (13) are found as

$$
\begin{aligned}
C_{1} & =\frac{2\left(\lambda_{1}+3\right) \Omega a^{\xi} b^{\frac{1}{2}(\beta+\xi+6)}-2\left(\lambda_{1}+3\right) \Omega b^{\xi} a^{\frac{1}{2}(\beta+\xi+6)}}{\left(a^{\xi}-b^{\xi}\right)\left(\beta-2 \lambda_{1}+\xi\right)} \\
C_{2} & =\frac{2\left(\lambda_{1}+3\right) \Omega\left(a^{\frac{1}{2}(\beta+\xi+6)}-b^{\frac{1}{2}(\beta+\xi+6)}\right)}{\left(a^{\xi}-b^{\xi}\right)\left(\beta-2 \lambda_{1}-\xi\right)}
\end{aligned}
$$

Substitution of Equation (14) into Equations (11) and (13) gives the explicit form of the radial displacement, the radial stress and the tangential stress as follows, respectively.

$$
\begin{gathered}
u_{r}(\mathrm{r})=\frac{\Omega r^{\frac{1}{2}(-\beta-\xi)}}{\left(a^{\xi}-b^{\xi}\right)\left(\beta-2 \lambda_{1}+\xi\right)\left(-\beta+2 \lambda_{1}+\xi\right)}\left(2\left(\lambda_{1}+3\right) a^{\xi / 2} b^{\xi / 2}(-\beta\right. \\
\left.+2 \lambda_{1}+\xi\right)\left(a^{\xi / 2} b^{\frac{\beta}{2}+3}-a^{\frac{\beta}{2}+3} b^{\xi / 2}\right) \\
-2\left(\lambda_{1}+3\right) r^{\xi}\left(\beta-2 \lambda_{1}+\xi\right)\left(a^{\frac{1}{2}(\beta+\xi+6)}-b^{\frac{1}{2}(\beta+\xi+6)}\right) \\
\left.+\left(a^{\xi}-b^{\xi}\right)\left(\beta-2 \lambda_{1}+\xi\right)\left(-\beta+2 \lambda_{1}+\xi\right) r^{\frac{1}{2}(\beta+\xi+6)}\right) \\
\sigma_{r}(r)=-\frac{1}{a^{\xi}-b^{\xi}}\left(C _ { 0 } ( \lambda _ { 1 } + 3 ) \Omega ( \frac { r } { b } ) ^ { \beta } r ^ { \frac { 1 } { 2 } ( - \beta - \xi - 2 ) } \left(a^{\frac{1}{2}(\beta+\xi+6)}\left(r^{\xi}-b^{\xi}\right)\right.\right. \\
+a^{\xi}\left(b^{\frac{1}{2}(\beta+\xi+6)}-r^{\frac{1}{2}(\beta+\xi+6)}\right)-r^{\xi} b^{\frac{1}{2}(\beta+\xi+6)} \\
\left.\left.+b^{\xi} r^{\frac{1}{2}(\beta+\xi+6)}\right)\right)
\end{gathered}
$$




$$
\begin{aligned}
& \sigma_{\theta}(r) \\
& =\frac{1}{\left(a^{\xi}-b^{\xi}\right)\left(\beta-2 \lambda_{1}-\xi\right)\left(\beta-2 \lambda_{1}+\xi\right)}\left\{C _ { 0 } \Omega ( \frac { r } { b } ) ^ { \beta } r ^ { \frac { 1 } { 2 } ( - \beta - \xi - 2 ) } \left(\lambda _ { 1 } \left(a ^ { \xi / 2 } b ^ { \xi / 2 } \left(2 \lambda_{2}(\beta\right.\right.\right.\right. \\
& -\xi-6)-3(\beta-\xi)(\beta+\xi))\left(a^{\xi / 2} b^{\frac{\beta}{2}+3}-a^{\frac{\beta}{2}+3} b^{\xi / 2}\right) \\
& -r^{\xi}\left(3(\beta-\xi)(\beta+\xi)-2 \lambda_{2}(\beta+\xi-6)\right)\left(a^{\frac{1}{2}(\beta+\xi+6)}-b^{\frac{1}{2}(\beta+\xi+6)}\right) \\
& \left.-\left(a^{\xi}-b^{\xi}\right)\left(-3 \beta^{2}+4 \beta \lambda_{2}+3 \xi^{2}\right) r^{\frac{1}{2}(\beta+\xi+6)}\right) \\
& +\lambda_{2}\left(6(\beta+\xi) r^{\xi}\left(a^{\frac{1}{2}(\beta+\xi+6)}-b^{\frac{1}{2}(\beta+\xi+6)}\right)-6 b^{\xi}(\beta-\xi) a^{\frac{1}{2}(\beta+\xi+6)}\right. \\
& \left.-6 a^{\xi}(\xi-\beta) b^{\frac{1}{2}(\beta+\xi+6)}-\left(\xi^{2}-\beta^{2}\right)\left(a^{\xi}-b^{\xi}\right) r^{\frac{1}{2}(\beta+\xi+6)}\right) \\
& +2 \lambda_{1}^{3}\left(-a^{\frac{1}{2}(\beta+\xi+6)}\left((\beta+\xi) b^{\xi}+(\xi-\beta) r^{\xi}\right)\right. \\
& +a^{\xi}\left((\beta+\xi) b^{\frac{1}{2}(\beta+\xi+6)}+6 r^{\frac{1}{2}(\beta+\xi+6)}\right)+(\xi-\beta) r^{\xi} b^{\frac{1}{2}(\beta+\xi+6)} \\
& \left.-6 b^{\xi} r^{\frac{1}{2}(\beta+\xi+6)}\right) \\
& +\lambda_{1}^{2}\left(a^{\xi / 2} b^{\xi / 2}\left(-(\beta-\xi-6)(\beta+\xi)-4 \lambda_{2}\right)\left(a^{\frac{1}{\xi} / 2} b^{\frac{\beta}{2}+3}-a^{\frac{\beta}{2}+3} b^{\xi / 2}\right)\right. \\
& -r^{\xi}\left((\beta-\xi)(\beta+\xi-6)+4 \lambda_{2}\right)\left(a^{\frac{1}{2}(\beta+\xi+6)}-b^{\frac{1}{2}(\beta+\xi+6)}\right) \\
& \left.\left.\left.-4\left(3 \beta-\lambda_{2}\right)\left(a^{\xi}-b^{\xi}\right) r^{\frac{1}{2}(\beta+\xi+6)}\right)\right)\right\}
\end{aligned}
$$

\section{Verification of the Formulas}

As a comparative example, a stress-free disc with $a=2 \mathrm{~cm}$, and $b=5 \mathrm{~cm}$ $(a / b=0.4)$ is chosen. The disk is made of an injection molded Nylon 6 composite containing 40wt\% short glass fiber. Material properties of the disc are given in Table 1.

Table 1. Material Properties

\begin{tabular}{|l|c|c|c|c|}
\hline & $\begin{array}{c}E_{r} \\
(\mathrm{GPa})\end{array}$ & $\begin{array}{c}E_{\theta} \\
(\mathrm{GPa})\end{array}$ & $\rho\left(\frac{\mathrm{kg}}{\mathrm{m}^{3}}\right)$ & $v_{r \theta}$ \\
\hline $\begin{array}{l}\text { An injection molded Nylon 6 composite } \\
\text { containing 40wt\% short glass fiber }\end{array}$ & 12.0 & 20.0 & 1600 & 0.21 \\
\hline
\end{tabular}

Dimensionless elastic stress and displacements are defined as

$\bar{\sigma}_{r}=\frac{\sigma_{\mathrm{r}}}{\rho_{b} \omega^{2} b^{2}}$ 


$$
\begin{aligned}
& \bar{\sigma}_{\theta}=\frac{\sigma_{\theta}}{\rho_{b} \omega^{2} b^{2}} \\
& \bar{u}_{r}=\frac{\mathrm{E}_{\theta}}{\rho_{b} \omega^{2} b^{3}} u_{r}
\end{aligned}
$$

Comparisons of dimensionless radial displacements, radial and hoop stresses with the open literature for $\beta=-1$ are given in Table 2. A perfect harmony is observed among the results.

Table 2. Comparisons of Dimensionless Radial Displacement, Radial Stress, and Hoop Stress with the Open Literature for $\beta=-1$

\begin{tabular}{|c|c|c|c|c|}
\hline $\mathrm{a} / \mathrm{b}$ & $\begin{array}{c}\text { Exact } \\
\text { (Present Equation [16]) }\end{array}$ & $\begin{array}{c}\text { Exact } \\
\text { (Peng and Li 2012) }\end{array}$ & $\begin{array}{c}\text { Numerical } \\
\text { Peng and Li } \\
2012)\end{array}$ & $\begin{array}{c}\text { Numerical } \\
\text { (Boğa 2016) }\end{array}$ \\
\hline & & $\bar{u}_{r}$ & & \\
\hline 0.4 & 0.26783 & 0.2678 & 0.2678 & 0.2678293 \\
\hline 0.5 & 0.255258 & 0.2553 & 0.2554 & 0.2552581 \\
\hline 0.6 & 0.256099 & 0.2561 & 0.2563 & 0.2560991 \\
\hline 0.7 & 0.262237 & 0.2622 & 0.2625 & 0.2622363 \\
\hline 0.8 & 0.268456 & 0.2685 & 0.2686 & 0.2684556 \\
\hline 0.9 & 0.270831 & 0.2708 & 0.2709 & 0.2708311 \\
\hline 1 & 0.26608 & 0.2661 & 0.2661 & 0.2660798 \\
\hline & & $\bar{\sigma}_{r}$ & & \\
\hline 0.4 & 0 & 0 & 0 & 0 \\
\hline 0.5 & 0.177997 & 0.1780 & 0.1781 & 0.1779964 \\
\hline 0.6 & 0.21002 & 0.2100 & 0.2099 & 0.2100199 \\
\hline 0.7 & 0.185223 & 0.1852 & 0.1856 & 0.1852227 \\
\hline 0.8 & 0.134617 & 0.1346 & 0.1349 & 0.1346162 \\
\hline 0.9 & 0.0709175 & 0.0709 & 0.0711 & 0.07091725 \\
\hline 1 & 0 & 0 & 0 & 0 \\
\hline & & $\bar{\sigma}_{\theta}$ & & \\
\hline 0.4 & 1.67393 & 1.6739 & 1.6739 & 1.673933 \\
\hline 0.5 & 1.08333 & 1.0833 & 1.0825 & 1.083331 \\
\hline 0.6 & 0.784894 & 0.7849 & 0.7839 & 0.7848933 \\
\hline 0.7 & 0.600005 & 0.6000 & 0.6000 & 0.6000040 \\
\hline 0.8 & 0.466578 & 0.4666 & 0.4663 & 0.466577 \\
\hline 0.9 & 0.359181 & 0.3592 & 0.3596 & 0.3591804 \\
\hline 1 & 0.26608 & 0.2661 & 0.2661 & 0.2660798 \\
\hline & & & & \\
\hline & & & & \\
\hline & & & & \\
\hline
\end{tabular}


Table 3. Variation of Elastic Fields with the Inhomogeneity Index and $(a / b)$

\begin{tabular}{|c|c|c|c|c|c|c|c|}
\hline$a / b$ & $\beta=-5$ & $\beta=-3$ & $\beta=-1$ & $\beta=0$ & $\beta=1$ & $\beta=3$ & $\beta=5$ \\
\hline \multicolumn{8}{|c|}{$\bar{u}_{r}$} \\
\hline 0.4 & 0.133642 & 0.180766 & 0.26783 & 0.329539 & 0.401457 & 0.558106 & 0.701461 \\
\hline 0.5 & 0.126625 & 0.171941 & 0.255258 & 0.314079 & 0.382424 & 0.530565 & 0.665171 \\
\hline 0.6 & 0.126949 & 0.173079 & 0.256099 & 0.31383 & 0.380229 & 0.52205 & 0.64858 \\
\hline 0.7 & 0.132142 & 0.179552 & 0.262237 & 0.318607 & 0.382669 & 0.517389 & 0.635578 \\
\hline 0.8 & 0.139548 & 0.187396 & 0.268456 & 0.322817 & 0.384034 & 0.511388 & 0.621949 \\
\hline 0.9 & 0.145661 & 0.192619 & 0.270831 & 0.322827 & 0.38112 & 0.501804 & 0.606124 \\
\hline 1 & 0.145648 & 0.190927 & 0.26608 & 0.31596 & 0.371837 & 0.487428 & 0.58728 \\
\hline \multicolumn{8}{|c|}{$\bar{\sigma}_{r}$} \\
\hline 0.4 & 0. & 0 . & 0 . & 0. & 0. & 0. & 0. \\
\hline 0.5 & 1.23574 & 0.470408 & 0.177997 & 0.106551 & 0.0618906 & 0.0188078 & 0.00504674 \\
\hline 0.6 & 0.879664 & 0.437232 & 0.21002 & 0.140871 & 0.0916517 & 0.0352044 & 0.0121716 \\
\hline 0.7 & 0.516414 & 0.316927 & 0.185223 & 0.136928 & 0.0984123 & 0.0467708 & 0.0205031 \\
\hline 0.8 & 0.267712 & 0.194997 & 0.134617 & 0.108397 & 0.0851786 & 0.0491921 & 0.0268568 \\
\hline 0.9 & 0.105838 & 0.0888756 & 0.0709175 & 0.0616511 & 0.0525424 & 0.0363096 & 0.0242649 \\
\hline 1 & 0. & 0 . & 0. & 0. & 0. & 0. & 0 . \\
\hline \multicolumn{8}{|c|}{$\bar{\sigma}_{\theta}$} \\
\hline 0.4 & 32.6275 & 7.06116 & 1.67393 & 0.823848 & 0.401457 & 0.089297 & 0.0179574 \\
\hline 0.5 & 8.5365 & 2.9157 & 1.08333 & 0.66545 & 0.404085 & 0.139224 & 0.0433395 \\
\hline 0.6 & 3.02885 & 1.48851 & 0.784894 & 0.572355 & 0.412307 & 0.20026 & 0.088316 \\
\hline 0.7 & 1.30393 & 0.858746 & 0.600005 & 0.503078 & 0.417113 & 0.269891 & 0.159778 \\
\hline 0.8 & 0.626033 & 0.525758 & 0.466578 & 0.44146 & 0.413846 & 0.344506 & 0.26415 \\
\hline 0.9 & 0.31113 & 0.324688 & 0.359181 & 0.380275 & 0.39951 & 0.41917 & 0.406171 \\
\hline 1 & 0.145648 & 0.190927 & 0.26608 & 0.31596 & 0.371837 & 0.487428 & 0.58728 \\
\hline
\end{tabular}

Variations of the dimensionless radial displacement, radial and hoop stresses along the radial direction for different inhomogeneity indexes are presented in Table 3. As observed from Table 3, variation of the inhomogeneity indexes from $\beta=5$ through $\beta=-5$ makes the radial dimensionless displacement small. However when the inhomogeneity indexes are changed from positive to the negative both radial and hoop stresses become much higher.

\section{Numerical Examples}

In this section it is aimed to study the thickness effects on the variation of the radial displacement, the radial stress and the hoop stress. The outer radius of the test annulus is determined as $b=10 \mathrm{~cm}=$ constant. The inner radius of the annulus varies from $a=2 \mathrm{~cm}$ to $a=9.95 \mathrm{~cm}$. The same material properties presented in Table 1 are used in the parametric study:

Numerical results are illustrated in Figures 2-6 for $a=2 \mathrm{~cm}, a=4 \mathrm{~cm}, a=6 \mathrm{~cm}$, $a=8 \mathrm{~cm}$, and $a=9.95 \mathrm{~cm}$, and presented in Tables 4 and 5 for $a=3 \mathrm{~cm}, a=5 \mathrm{~cm}$, $a=7 \mathrm{~cm}$, and $a=9 \mathrm{~cm}$. In these Figures and Tables inhomogeneity indexes are chosen as to be in the range of $-6 \leq \beta \leq 6$.

Some results are outlined below:

- Increasing the aspect ratio from 0.2 toward 0.995 results in a magnification in the radial displacements.

- Negative inhomogeneity indexes offer smaller radial displacements. 
- For a very thin annulus, that is for a ring, the effect of the inhomogeneity index on the radial displacement and the radial stress almost disappears. If it is small, however, it has still effect to some extent on the tangential stresses.

- Maximum radial stresses decrease with increasing aspect ratio from the thick annulus to the rings.

- Positive inhomogeneity indexes offer more small radial stresses.

- Positive inhomogeneity indexes also offer smaller hoop stresses.

- Centrifugal force-induced stresses are completely in tension for all inhomogeneity indexes and aspect ratios.

- Negative inhomogeneity parameters present more noticeable hoop stresses at the inner surface of the annulus.

- At the outer surface, positive inhomogeneity indexes have higher hoop stresses for higher aspect ratios that is for thinner annulus.

\section{Discussion and Conclusions}

In this study, a circular annulus rotating at a constant angular velocity and made of such functionally graded polar orthotropic materials is handled analytically. Navier equation in terms of the radial displacement is derived from the elasticity theory under axisymmetric plane stress assumption. To get a closedform solution, a simple power material grading rule is chosen. By doing so, the governing equation turns into a second order differential equation with constant coefficients at which Euler-Cauchy solution technique may be applied. The centrifugal force is added to the equilibrium equation in the radial direction as a body force.

After getting both the homogeneous and particular analytical solutions to the problem, a parametric study is accomplished. The ratio of the inner and outer radius of the annulus is defined as the aspect ratio. The effect of the aspect ratio of a rotating circular annulus with uniform thickness on the dimensionless radial displacement, the dimensionless radial stress and tangential stress is investigated. The aspect ratio of the annulus is assumed to be changed from 0.2 towards 0.995 for rings, while the inhomogeneity parameter is changing between -6 and 6 .

It is observed that both the aspect ratio and inhomogeneity index have significant impact on the linear elastic response arising out of rotation of the annulus at a constant angular velocity. Increasing aspect ratio from 0.2 toward 0.995 causes a magnification in the radial displacements. The effect of the inhomogeneity index on the radial displacement and the radial stress almost disappears while it has still effect to some extent on the tangential stresses. 
Table 4. Variation of Elastic Fields with $\beta=-6,-4,-2,0,2,4,6$

\begin{tabular}{|c|c|c|c|c|c|c|c|}
\hline$r-\mathrm{m}$ & $\beta=-6$ & $\beta=-4$ & $\beta=-2$ & $\beta=0$ & $\beta=2$ & $\beta=4$ & $\beta=6$ \\
\hline \multicolumn{8}{|c|}{$a / b=0.3 ; \quad \bar{u}_{r}$} \\
\hline .03 & 0.051638 & 0.070893 & 0.120113 & 0.233505 & 0.418588 & 0.61716 & 0.778835 \\
\hline .04 & 0.048640 & 0.067417 & 0.114984 & 0.223366 & 0.398153 & 0.58322 & 0.73177 \\
\hline .06 & 0.056645 & 0.080338 & 0.134179 & 0.244985 & 0.409349 & 0.572028 & 0.695967 \\
\hline .07 & 0.065729 & 0.092327 & 0.148718 & 0.258519 & 0.415352 & 0.566822 & 0.680534 \\
\hline .09 & 0.085070 & 0.1138 & 0.169648 & 0.272012 & 0.413086 & 0.546648 & 0.645885 \\
\hline .10 & 0.087482 & 0.11534 & 0.169143 & 0.267391 & 0.402542 & 0.530384 & 0.625336 \\
\hline \multicolumn{8}{|c|}{$a / b=0.3 ; \quad \bar{\sigma}_{r}$} \\
\hline .04 & 6.2492 & 1.66112 & 0.486923 & 0.139081 & 0.032988 & 0.006241 & 0.001016 \\
\hline .05 & 3.1827 & 1.22138 & 0.488501 & 0.183234 & 0.057105 & 0.014619 & 0.003344 \\
\hline .06 & 1.53486 & 0.778176 & 0.395727 & 0.185284 & 0.073101 & 0.024557 & 0.007648 \\
\hline .07 & 0.73917 & 0.465808 & 0.287582 & 0.16256 & 0.079030 & 0.033898 & 0.0139 \\
\hline .08 & 0.336856 & 0.252518 & 0.183256 & 0.122133 & 0.071661 & 0.038334 & 0.020092 \\
\hline .09 & 0.121347 & 0.104739 & 0.087255 & 0.067354 & 0.046911 & 0.030678 & 0.020046 \\
\hline \multicolumn{8}{|c|}{$a / b=0.3 ; \quad \bar{\sigma}_{\theta}$} \\
\hline .03 & 236.113 & 29.1739 & 4.44862 & 0.778351 & 0.125576 & 0.016663 & 0.001893 \\
\hline .04 & 31.8744 & 7.16504 & 1.96705 & 0.607093 & 0.170807 & 0.039511 & 0.007849 \\
\hline .05 & 7.59394 & 2.70986 & 1.14372 & 0.526756 & 0.220776 & 0.077096 & 0.023412 \\
\hline .06 & 2.56071 & 1.30551 & 0.759704 & 0.473158 & 0.271195 & 0.132153 & 0.056795 \\
\hline .07 & 1.05683 & 0.712368 & 0.534233 & 0.426208 & 0.318407 & 0.206284 & 0.119242 \\
\hline .08 & 0.48122 & 0.407713 & 0.379926 & 0.378213 & 0.358722 & 0.29943 & 0.224643 \\
\hline .09 & 0.22033 & 0.229379 & 0.263252 & 0.32581 & 0.388197 & 0.409244 & 0.388405 \\
\hline .10 & 0.08748 & 0.11534 & 0.169143 & 0.267391 & 0.402542 & 0.530384 & 0.625336 \\
\hline \multicolumn{8}{|c|}{$a / b=0.5 ; \quad \bar{u}_{r}$} \\
\hline .05 & 0.22403 & 0.267705 & 0.335178 & 0.429083 & 0.541008 & 0.65368 & 0.752386 \\
\hline .06 & 0.21294 & 0.254947 & 0.319673 & 0.40949 & 0.516192 & 0.623205 & 0.71656 \\
\hline .07 & 0.20905 & 0.250899 & 0.314643 & 0.402101 & 0.504862 & 0.606832 & 0.694883 \\
\hline .08 & 0.20949 & 0.251241 & 0.313812 & 0.398467 & 0.496773 & 0.593367 & 0.6761 \\
\hline .09 & 0.21074 & 0.251643 & 0.31222 & 0.393435 & 0.487114 & 0.578705 & 0.656869 \\
\hline .10 & 0.20777 & 0.247226 & 0.305487 & 0.383446 & 0.473253 & 0.560985 & 0.635813 \\
\hline \multicolumn{8}{|c|}{$a / b=0.5 ; \quad \bar{\sigma}_{r}$} \\
\hline .06 & 0.749997 & 0.361178 & 0.172664 & 0.079158 & 0.033810 & 0.013339 & 0.004919 \\
\hline .07 & 0.508027 & 0.307171 & 0.181099 & 0.101314 & 0.052744 & 0.025548 & 0.011718 \\
\hline .08 & 0.264157 & 0.191608 & 0.134379 & 0.089312 & 0.055623 & 0.032676 & 0.018487 \\
\hline .09 & 0.101571 & 0.085427 & 0.069409 & 0.053726 & 0.039434 & 0.027754 & 0.019138 \\
\hline \multicolumn{8}{|c|}{$a / b=0.5 ; \quad \bar{\sigma}_{\theta}$} \\
\hline .05 & 28.6757 & 8.56657 & 2.68142 & 0.858166 & 0.270504 & 0.08171 & 0.023512 \\
\hline .06 & 7.86928 & 3.40506 & 1.5404 & 0.710189 & 0.321549 & 0.139281 & 0.057441 \\
\hline .07 & 2.71624 & 1.60033 & 0.980712 & 0.60989 & 0.371864 & 0.217085 & 0.12089 \\
\hline .08 & 1.09138 & 0.833789 & 0.659946 & 0.529343 & 0.416886 & 0.31524 & 0.228015 \\
\hline .09 & 0.476145 & 0.456059 & 0.452578 & 0.455954 & 0.452204 & 0.43159 & 0.394573 \\
\hline .10 & 0.207771 & 0.247226 & 0.305487 & 0.383446 & 0.473253 & 0.560985 & 0.635813 \\
\hline \multicolumn{8}{|c|}{$a / b=0.7 ; \quad \bar{u}_{r}$} \\
\hline .07 & 0.524053 & 0.557942 & 0.596839 & 0.639616 & 0.684468 & 0.729245 & 0.77193 \\
\hline .08 & 0.502458 & 0.535108 & 0.572543 & 0.613669 & 0.656738 & 0.699681 & 0.740564 \\
\hline .09 & 0.486931 & 0.51855 & 0.554696 & 0.594294 & 0.635652 & 0.676785 & 0.715848 \\
\hline .10 & 0.472149 & 0.502599 & 0.53737 & 0.575425 & 0.615138 & 0.654608 & 0.692069 \\
\hline \multicolumn{8}{|c|}{$a / b=0.7 ; \quad \bar{\sigma}_{r}$} \\
\hline .08 & 0.104376 & 0.073666 & 0.051252 & 0.035020 & 0.023443 & 0.015365 & 0.009870 \\
\hline .09 & 0.058106 & 0.0480345 & 0.0390588 & 0.0311825 & 0.0244304 & 0.0188055 & 0.0142602 \\
\hline \multicolumn{8}{|c|}{$a / b=0.7 ; \quad \bar{\sigma}_{\theta}$} \\
\hline .07 & 6.36339 & 3.3197 & 1.74005 & 0.913738 & 0.479128 & 0.250131 & 0.129738 \\
\hline .08 & 2.43244 & 1.6588 & 1.13619 & 0.779343 & 0.533595 & 0.363615 & 0.246122 \\
\hline .09 & 1.03839 & 0.894981 & 0.774571 & 0.67124 & 0.580637 & 0.499958 & 0.427692 \\
\hline .10 & 0.472149 & 0.502599 & 0.53737 & 0.575425 & 0.615138 & 0.654608 & 0.692069 \\
\hline \multicolumn{8}{|c|}{$a / b=0.9 ; \quad \bar{u}_{r}$} \\
\hline
\end{tabular}




\begin{tabular}{|l|r|r|r|r|r|r|r|}
\hline .09 & 0.85616 & 0.861416 & 0.866746 & 0.872121 & 0.877518 & 0.882913 & 0.888282 \\
\hline .10 & 0.825576 & 0.830649 & 0.835787 & 0.840969 & 0.846171 & 0.85137 & 0.856545 \\
\hline \multicolumn{8}{|c|}{$a / b=0.9 ; \quad \bar{\sigma}_{\theta}$} \\
\hline .09 & 1.79001 & 1.45882 & 1.18895 & 0.969023 & 0.789766 & 0.643643 & 0.524522 \\
\hline .10 & 0.825576 & 0.830649 & 0.835787 & 0.840969 & 0.846171 & 0.85137 & 0.856545 \\
\hline
\end{tabular}

Table 5. Variation of Elastic Fields with $\beta=-5,-3,-1,0,1,3,5$

\begin{tabular}{|c|c|c|c|c|c|c|c|}
\hline$r-m$ & $\beta=-5$ & $\beta=-3$ & $\beta=-1$ & $\beta=0$ & $\beta=1$ & $\beta=3$ & $\beta=5$ \\
\hline \multicolumn{8}{|c|}{$a / b=0.3 ; \quad \bar{u}_{r}$} \\
\hline .03 & 0.059117 & 0.0898232 & 0.166765 & 0.233505 & 0.319633 & 0.520584 & 0.703657 \\
\hline .04 & 0.0559492 & 0.0857695 & 0.15973 & 0.223366 & 0.304983 & 0.493538 & 0.662963 \\
\hline .06 & 0.0661576 & 0.101819 & 0.181217 & 0.244985 & 0.323319 & 0.494433 & 0.639235 \\
\hline .07 & 0.0766369 & 0.115269 & 0.19597 & 0.258519 & 0.333875 & 0.494939 & 0.628632 \\
\hline .09 & 0.0971885 & 0.137037 & 0.214307 & 0.272012 & 0.340275 & 0.483503 & 0.600673 \\
\hline .10 & 0.0992596 & 0.137761 & 0.212039 & 0.267391 & 0.332809 & 0.469952 & 0.582079 \\
\hline \multicolumn{8}{|c|}{$a / b=0.3 ; \quad \bar{\sigma}_{r}$} \\
\hline .04 & 3.17189 & 0.893102 & 0.263997 & 0.139081 & 0.0697796 & 0.0147079 & 0.002551 \\
\hline .05 & 1.95422 & 0.772259 & 0.304249 & 0.183234 & 0.105131 & 0.0294793 & 0.007051 \\
\hline .06 & 1.08881 & 0.557069 & 0.275448 & 0.185284 & 0.119179 & 0.0430143 & 0.0137655 \\
\hline .07 & 0.586635 & 0.36821 & 0.219739 & 0.16256 & 0.115608 & 0.0523087 & 0.0217375 \\
\hline .08 & 0.292173 & 0.216587 & 0.151788 & 0.122133 & 0.0950424 & 0.0527636 & 0.0277305 \\
\hline .09 & 0.113032 & 0.0962325 & 0.0776141 & 0.0673544 & 0.0568893 & 0.0380657 & 0.0247382 \\
\hline \multicolumn{8}{|c|}{$a / b=0.3 ; \quad \bar{\sigma}_{\theta}$} \\
\hline .03 & 81.0933 & 11.0893 & 1.85294 & 0.778351 & 0.319633 & 0.0468526 & 0.0056996 \\
\hline .04 & 14.7696 & 3.66296 & 1.09071 & 0.607093 & 0.329406 & 0.0841139 & 0.0178648 \\
\hline .05 & 4.44563 & 1.72605 & 0.776294 & 0.526756 & 0.348404 & 0.133342 & 0.0430442 \\
\hline .06 & 1.79907 & 0.980615 & 0.599787 & 0.473158 & 0.365031 & 0.193051 & 0.0876627 \\
\hline .07 & 0.856725 & 0.608961 & 0.476847 & 0.426208 & 0.374338 & 0.260828 & 0.158543 \\
\hline .08 & 0.438212 & 0.388772 & 0.378166 & 0.378213 & 0.373431 & 0.333103 & 0.262026 \\
\hline .09 & 0.222439 & 0.242548 & 0.291741 & 0.32581 & 0.360187 & 0.40496 & 0.40276 \\
\hline .10 & 0.0992596 & 0.137761 & 0.212039 & 0.267391 & 0.332809 & 0.469952 & 0.582079 \\
\hline \multicolumn{8}{|c|}{$a / b=0.5 ; \quad \bar{u}_{r}$} \\
\hline .05 & 0.243321 & 0.298104 & 0.379052 & 0.429083 & 0.483783 & 0.598366 & 0.705333 \\
\hline .06 & 0.231508 & 0.284131 & 0.361672 & 0.40949 & 0.461684 & 0.570722 & 0.67211 \\
\hline .07 & 0.227606 & 0.279731 & 0.355663 & 0.402101 & 0.452508 & 0.556957 & 0.653068 \\
\hline .08 & 0.228092 & 0.279661 & 0.353653 & 0.398467 & 0.446822 & 0.54623 & 0.636886 \\
\hline .09 & 0.229031 & 0.279237 & 0.350522 & 0.393435 & 0.439582 & 0.534059 & 0.61985 \\
\hline .10 & 0.225433 & 0.273783 & 0.342269 & 0.383446 & 0.427698 & 0.518228 & 0.600378 \\
\hline \multicolumn{8}{|c|}{$a / b=0.5 ; \quad \bar{\sigma}_{r}$} \\
\hline .06 & 0.519942 & 0.250475 & 0.117776 & 0.0791583 & 0.0522513 & 0.0214402 & 0.0081585 \\
\hline .07 & 0.395639 & 0.237006 & 0.136561 & 0.101314 & 0.0737995 & 0.0370107 & 0.0173952 \\
\hline .08 & 0.22564 & 0.161351 & 0.110407 & 0.0893119 & 0.0710605 & 0.0428976 & 0.0246569 \\
\hline .09 & 0.0934648 & 0.0774105 & 0.0614763 & 0.0537263 & 0.0463205 & 0.0332147 & 0.0230732 \\
\hline \multicolumn{8}{|c|}{$a / b=0.5 ; \quad \bar{\sigma}_{\theta}$} \\
\hline .05 & 15.5725 & 4.76967 & 1.51621 & 0.858166 & 0.483783 & 0.149591 & 0.0440833 \\
\hline .06 & 5.14399 & 2.28003 & 1.04587 & 0.710189 & 0.479972 & 0.212964 & 0.0899609 \\
\hline .07 & 2.07309 & 1.24801 & 0.773638 & 0.60989 & 0.478338 & 0.285863 & 0.16289 \\
\hline .08 & 0.949075 & 0.73924 & 0.591226 & 0.529343 & 0.471693 & 0.364602 & 0.269498 \\
\hline .09 & 0.463674 & 0.452696 & 0.45426 & 0.455954 & 0.455794 & 0.444213 & 0.414759 \\
\hline .10 & 0.225433 & 0.273783 & 0.342269 & 0.383446 & 0.427698 & 0.518228 & 0.600378 \\
\hline \multicolumn{8}{|c|}{$a / b=0.7 ; \quad \bar{u}_{r}$} \\
\hline .07 & 0.540332 & 0.576817 & 0.617842 & 0.639616 & 0.661915 & 0.707001 & 0.750958 \\
\hline .08 & 0.518146 & 0.553278 & 0.592741 & 0.613669 & 0.635087 & 0.678355 & 0.720484 \\
\hline .09 & 0.502135 & 0.536108 & 0.574157 & 0.594294 & 0.614875 & 0.65637 & 0.696674 \\
\hline .10 & 0.486796 & 0.519494 & 0.556077 & 0.575425 & 0.595191 & 0.635022 & 0.673684 \\
\hline \multicolumn{8}{|c|}{$a / b=0.7 ; \quad \bar{\sigma}_{r}$} \\
\hline .08 & 0.0878205 & 0.0615715 & 0.0424692 & 0.0350196 & 0.0287284 & 0.0190286 & 0.0123439 \\
\hline
\end{tabular}




\begin{tabular}{|l|l|l|l|l|l|l|l|}
\hline .09 & 0.0529315 & 0.0434105 & 0.0349816 & 0.0311825 & 0.0276647 & 0.0214786 & 0.0164032 \\
\hline \multicolumn{8}{|c|}{$a / b=0.7 ; \quad \bar{\sigma}_{\theta}$} \\
\hline .07 & 4.59275 & 2.4024 & 1.2609 & 0.913738 & 0.661915 & 0.34643 & 0.180305 \\
\hline .08 & 2.00731 & 1.37233 & 0.941023 & 0.779343 & 0.645142 & 0.440807 & 0.299431 \\
\hline .09 & 0.963382 & 0.832307 & 0.72108 & 0.67124 & 0.624558 & 0.539177 & 0.462829 \\
\hline .10 & 0.486796 & 0.519494 & 0.556077 & 0.575425 & 0.595191 & 0.635022 & 0.673684 \\
\hline \multicolumn{8}{|c|}{$a / b=0.9 ; \bar{u}_{r}$} \\
\hline .09 & 0.858776 & 0.864074 & 0.869429 & 0.872121 & 0.874818 & 0.880217 & 0.885602 \\
\hline .10 & 0.828103 & 0.833212 & 0.838374 & 0.840969 & 0.843569 & 0.848772 & 0.853962 \\
\hline \multicolumn{8}{|c|}{$a / b=0.9 ; \quad \bar{\sigma}_{\theta}$} \\
\hline .09 & 1.61594 & 1.31698 & 1.07337 & 0.969023 & 0.874818 & 0.712976 & 0.581044 \\
\hline .10 & 0.828103 & 0.833212 & 0.838374 & 0.840969 & 0.843569 & 0.848772 & 0.853962 \\
\hline
\end{tabular}

Figure 2. Variation of the Dimensionless Radial Displacement with Inhomogeneity Indexes and Aspect Ratio
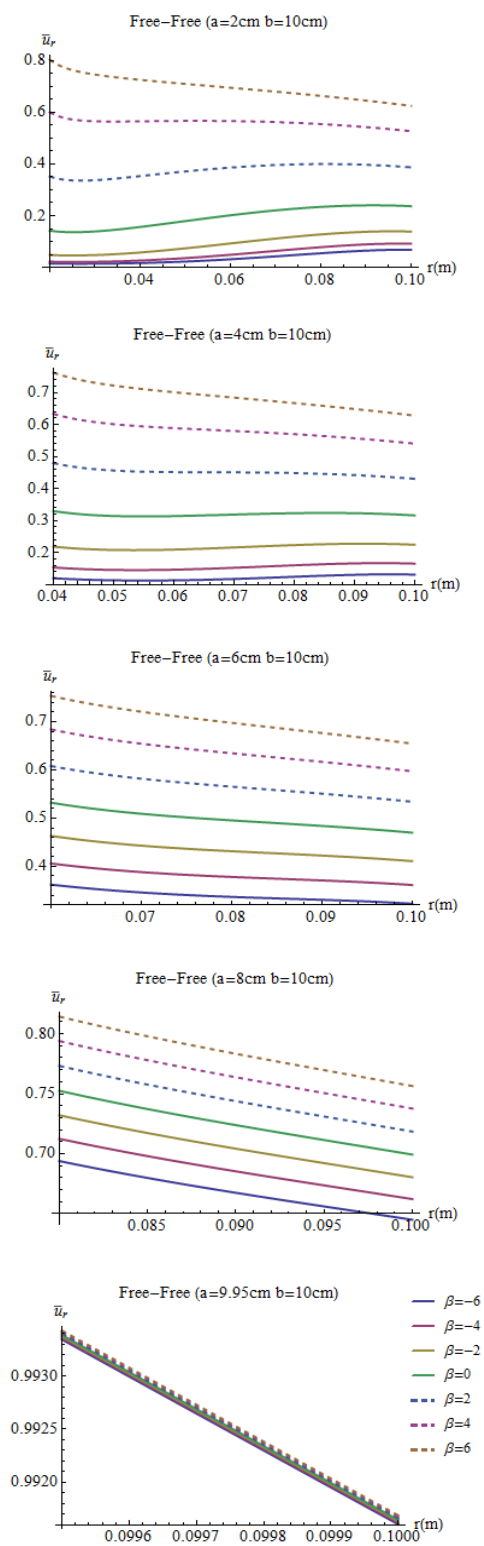
Figure 3. Variation of the Dimensionless Radial Stress with Inhomogeneity Indexes and Aspect Ratio
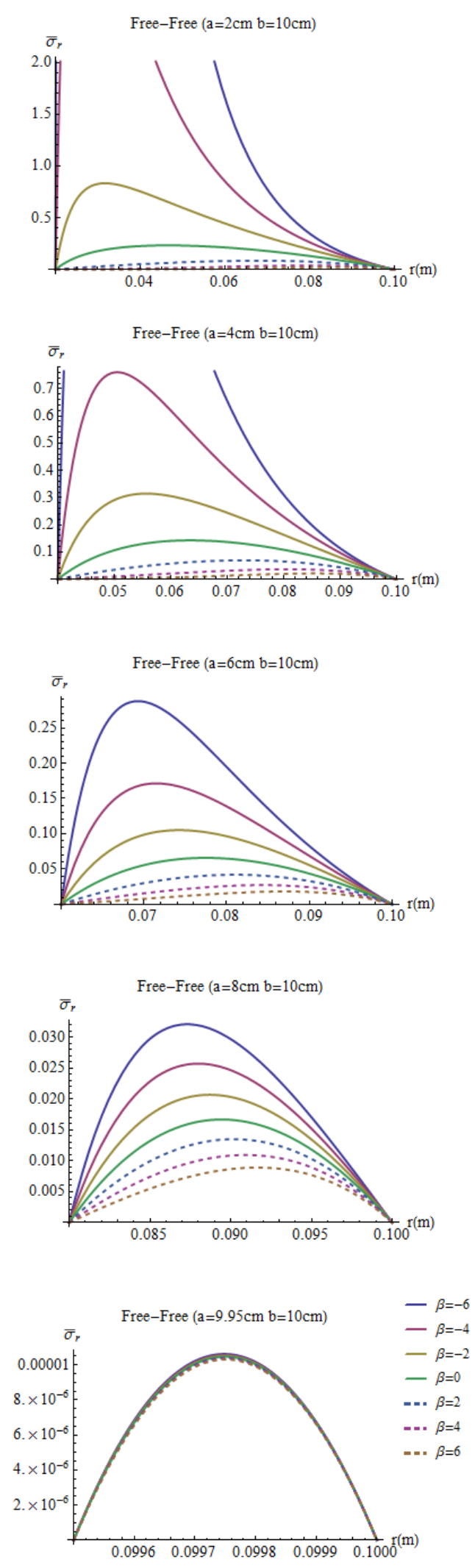
Figure 4. Variation of the Dimensionless Hoop Stress with Inhomogeneity Indexes and Aspect Ratio
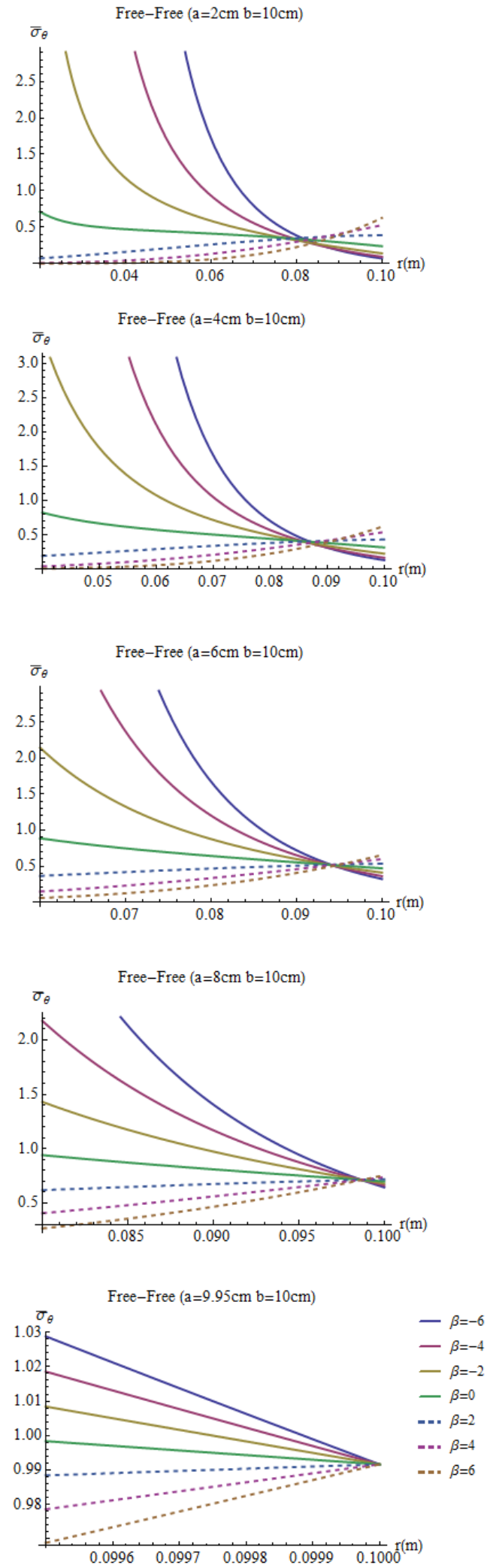
Figure 5. Variation of the Dimensionless Hoop Stress with Inhomogeneity Indexes and Aspect Ratio at the Inner Surface
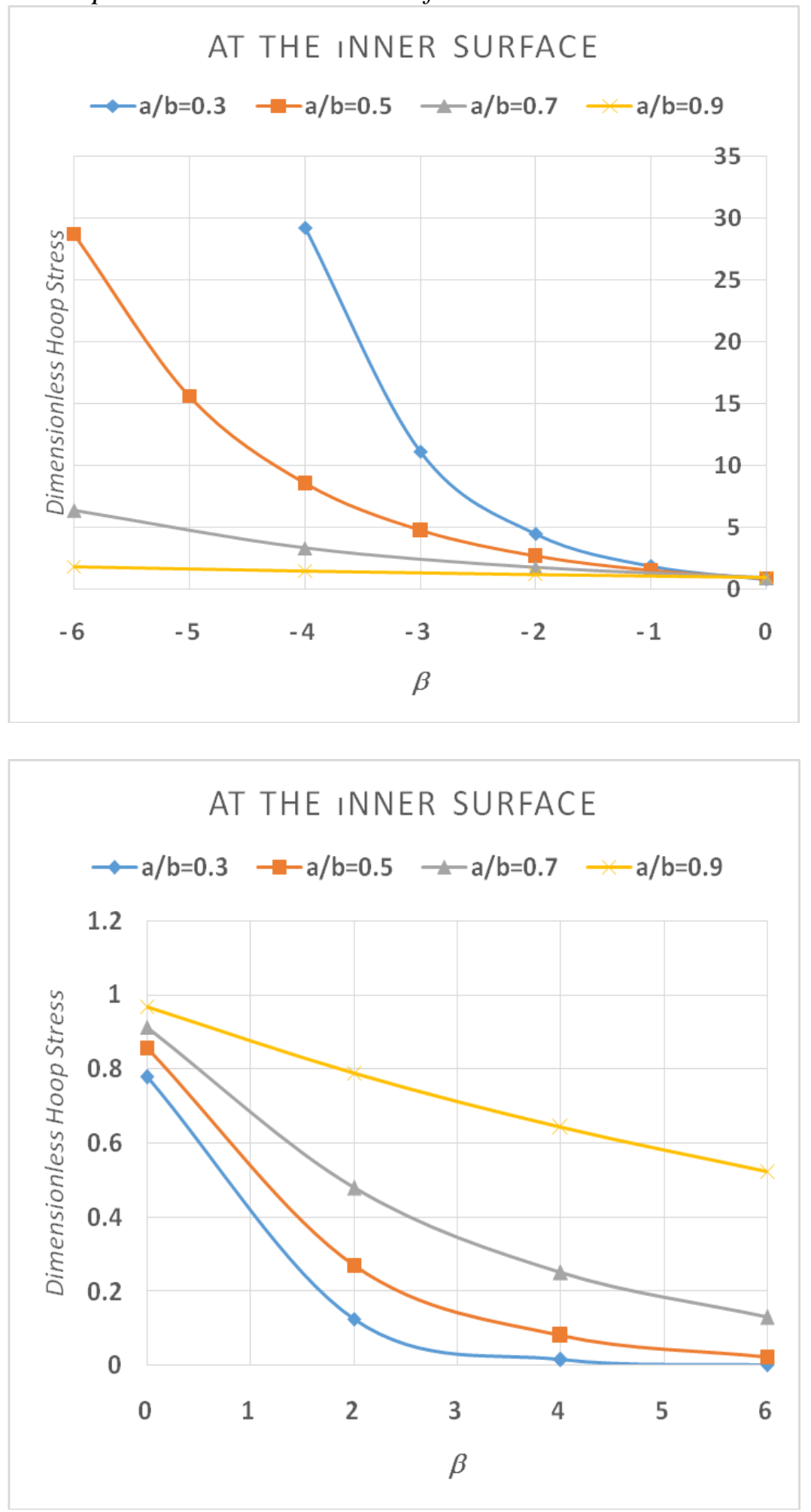
Figure 6. Variation of the Dimensionless Hoop Stress with Inhomogeneity Indexes and Aspect Ratio at the Outer Surface

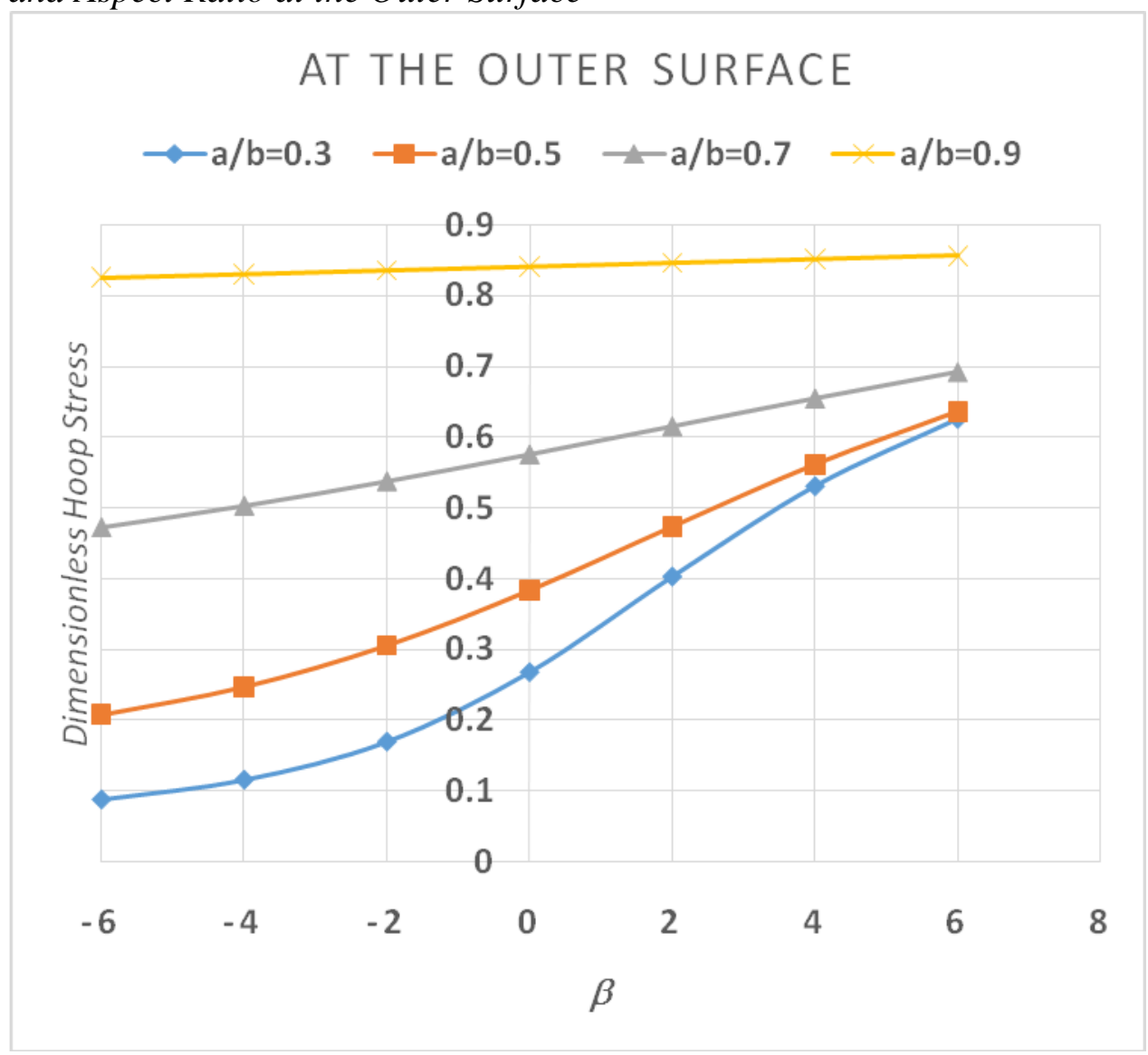

\section{Notations}

$\begin{array}{ll}a & \text { outer radius } \\ b & \text { inner radius } \\ C_{1}, C_{2} & \text { integration constants } \\ C_{i j} & \text { material on-axis rigidities } \\ E_{r}(r) & \text { Young's modulus in the } r \text { - direction } \\ E_{\theta}(r) & \text { Young's modulus in the } \theta \text { - direction } \\ E_{\theta}^{b} & \text { the reference value of Young's modulus of the mixture material } \\ & \text { located at the outer surface in the circumferential direction } \\ r & \text { radial direction } \\ u_{r} & \text { radial displacement } \\ \beta & \text { inhomogeneity index } \\ \varepsilon_{r} & \text { radial strain } \\ \varepsilon_{\theta} & \text { tangential strain } \\ \lambda_{2} & \text { anisotropy/polar degree of the material } \\ v_{\theta r}, v_{r \theta} & \text { anisotropic Poisson's ratios }\end{array}$




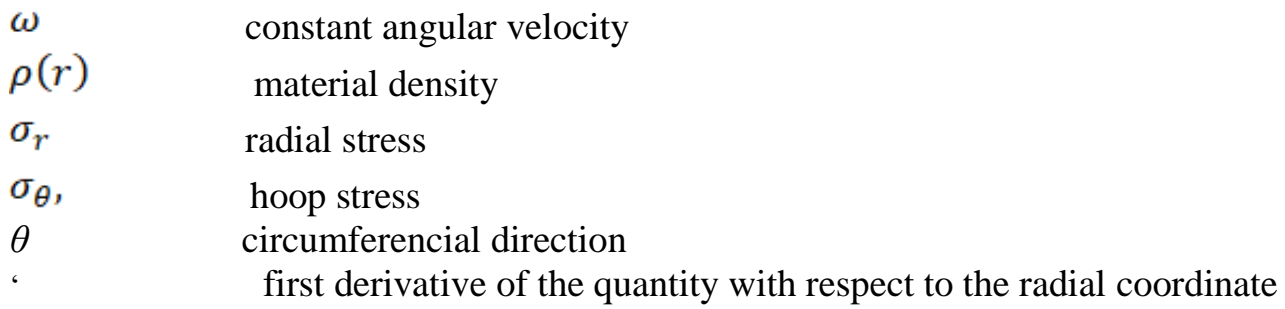

\section{References}

Ari-Gur J, Stavsky Y (1981) On rotating polar-orthotropic circular disks. International Journal of Solids and Structures 17(1): 57-67.

Boga C (2016) Analytical and numerical axisymmetric elastic stress analyses of stationary/rotating discs made of isotropic/orthotropic functionally graded materials by the transfer matrix method. Dissertation. Department of Mechanical Engineering, Çukurova University. No. 1698.

Çallığlu H (2007) Thermal stress analysis of curvilinearly orthotropic rotating discs. Journal of Thermoplastic Composite Materials 20(4): 357-369.

Çallığlu H, Topçu M, Altan G (2005) Stress analysis of curvilinearly orthotropic rotating discs under mechanical and thermal loading. Journal of Reinforced Plastics and Composites 24(8): 831-838.

Çallığlu H, Topçu M, Tarakçılar AR (2006) Elastic-plastic stress analysis of an orthotropic rotating disc. International Journal of Mechanical Sciences 48(9): 985990.

Campbell FC (2010) Structural Composite Materials. Ohio, USA: ASM International.

Chen J, Ding H, Chen W (2007) Three-dimensional analytical solution for a rotating disc of functionally graded materials with transverse isotropy. Archive of Applied Mechanics 77(4): 241-251.

Durodola J, Attia O (2000) Deformation and stresses in functionally graded rotating disks. Composites Science and Technology 60(7): 987-995.

Eraslan AN, Kaya Y, Varl E (2016) Analytical solutions to orthotropic variable thickness disk problems. Pamukkale University Journal of Engineering Sciences 22(1): 24-30.

Essa S, Argeso H (2017) Elastic analysis of variable profile and polar orthotropic FGM rotating disks for a variation function with three parameters. Acta Mechanica 228(11): 3877-3899.

Gopalakrishnan S, Chakraborty A, Roy Mahapatra D (2008) Spectral Finite Element Method. Wave Propagation, Diagnostics and Control in Anisotropic and Inhomogeneous Structures. London: Springer-Verlag.

Gupta U, Ansari A, Sharma S (2006) Buckling and vibration of polar orthotropic circular plate resting on Winkler foundation. Journal of Sound and Vibration 297(3-5): 457476.

Gurushankar GV (1975) Thermal stresses in a rotating, nonhomogeneous, anisotropic disk of varying thickness and density. The Journal of Strain Analysis for Engineering Design 10(3): 137-142.

Jain R, Ramachandra K, Simha KRY (1999) Rotating anisotropic disk of uniform strength. International Journal of Mechanical Sciences 41(6): 639-648.

Jain R, Ramachandra K, Simha KRY (2000) Singularity in rotating orthotropic disks and shells. International Journal of Solids and Structures 37(14): 2035-2038. 
Kacar I, Yildırım V (2017) Exact elastic analysis of functionally power-graded polar orthotropic rotating uniform disks under various boundary conditions. In $\mathrm{S}$ Sahinkaya, E Kalıpc1 (Eds.) Digital Proceeding of ICOCEE - Cappadocia 2017. Nevsehir, Turkey, May 8-10.

Koo KN (2006) Vibration analysis and critical speeds of polar orthotropic annular disks in rotation. Composite Structures 76(1): 67-72.

Koo KN (2008) Mechanical vibration and critical speeds of rotating composite laminate disks. Microsystem Technologies 14(6): 799-807.

Li SR, Batra RC, Ma LS (2007) Vibration of thermally post-buckled orthotropic circular plates. Journal of Thermal Stresses 30(1): 43-57.

Liang DS, Wang HJ, Chen LW (2002) Vibration and stability of rotating polar orthotropic annular disks subjected to a stationary concentrated transverse load. Journal of Sound and Vibration 250(5): 795-811.

Lubarda VA (2012) On pressurized curvilinearly orthotropic circular disk, cylinder and sphere made of radially nonuniform material. Journal of Elasticity 109(2): 103-133.

Mahamood RM, Akinlabi ET, Shukla M, Pityana S (2012) Functionally graded material: An overview. Proceedings of the World Congress on Engineering, WCE 2012, July 4-6. Volume III. London, U.K.

Mahamood RM, Akinlabi ET (2017) Functionally Graded Materials. Springer International Publishing.

Mian MA, Spencer A (1998) Exact solutions for functionally graded and laminated elastic materials. Journal of the Mechanics and Physics of Solids 46(12): 2283-2295.

Murthy D, Sherbourne A (1970) Elastic stresses in anisotropic disks of variable thickness. International Journal of Mechanical Sciences 12(7): 627-640.

Peng XL, Li XF (2012) Elastic analysis of rotating functionally graded polar orthotropic disks. International Journal of Mechanical Sciences 60(1): 84-91.

Reddy TY, Srinath H (1974) Elastic stresses in a rotating anisotropic annular disk of variable thickness and variable density. International Journal of Mechanical Sciences 16(2): 85-89.

Singh R, Jain S (2004) Free asymmetric transverse vibration of parabolically varying thickness polar orthotropic annular plate with flexible edge conditions. Journal of Science and Engineering 7(1): 41-52.

Tang S (1969) Elastic stresses in rotating anisotropic disks. International Journal of Mechanical Sciences 11(6): 509-517.

Tütüncü N (2000) Effect of anisotropy on stresses in rotating disks. International Journal of Mechanical Sciences 37(8): 873-881.

Wang X, Sudak LJ (2008) Three-dimensional analysis of multi-layered functionally graded anisotropic cylindrical panel under thermomechanical loading. Mechanics of Materials 40(4-5): 235-254.

Y1ldirım V (2018a) The Complementary Functions Method (CFM) Solution to the Elastic Analysis of Polar Orthotropic Rotating Discs. Journal of Applied and Computational Mechanics 4(3): 216-230.

Y1ldırım V (2018b) Unified exact solutions to the hyperbolically tapered pressurized/ rotating disks made of nonhomogeneous isotropic/orthotropic materials. International Journal of Advanced Materials Research 4(1): 1-23.

Yildırım V (2018c) The complementary functions method solution to the functionally graded polar orthotropic rotating hyperbolic disks with both radially and circumferentially aligned fibers. International Journal of Engineering \& Applied Sciences (IJEAS) 10(4): 276-290. 
Y1ldırım V (2018d) Numerical/analytical solutions to the elastic response of arbitrarily functionally graded polar orthotropic rotating discs. Journal of the Brazilian Society of Mechanical Sciences and Engineering 40/320(6): 1-21.

Zenkour AM (2009) Stress distribution in rotating composite structures of functionally graded solid disks. Journal of Materials Processing Technology 209(7): 3511-3517.

Zheng Y, Bahaloo H, Mousanezhad D, Vaziri A, Nayeb-Hashemi H (2017) Displacement and stress fields in a functionally graded fiber-reinforced rotating disk with nonuniformthickness and variable angular velocity. Journal of Engineering Materials and Technology 139(3): 031010-1-9. 
